Gerlach, Tobias; Harant, Jochen:

\title{
A note on domination in bipartite graphs
}

URN: $\quad$ urn:nbn:de:gbv:ilm1-2020200083

\begin{tabular}{|c|c|}
\hline Original published in: & $\begin{array}{l}\text { Discussiones mathematicae. Graph theory / Uniwersytet Zielonogórski, } \\
\text { Wydział Matematyki, Informatyki i Ekonometrii. - Warsaw : De Gruyter } \\
\text { Open. - } 22 \text { (2002), 2, p. 229-231. }\end{array}$ \\
\hline Original published: & 2002 \\
\hline ISSN: & $2083-5892$ \\
\hline DOI: & 10.7151/dmgt.1171 \\
\hline \multirow[t]{2}{*}{ [Visited: } & 2020-01-17] \\
\hline & $\begin{array}{l}\text { This work is licensed under a Creative Commons Attribution- } \\
\text { NonCommercial-NoDerivatives } 3.0 \text { Unported license. } \\
\text { To view a copy of this license, visit } \\
\text { http://creativecommons.org/licenses/BY-NC-ND/3.0/ }\end{array}$ \\
\hline
\end{tabular}


Discussiones Mathematicae

Graph Theory 22 (2002) 229-231

\title{
A NOTE ON DOMINATION IN BIPARTITE GRAPHS
}

\author{
Tobias Gerlach and Jochen Harant \\ Department of Mathematics \\ Technical University of Ilmenau \\ D-98684 Ilmenau, Germany
}

\begin{abstract}
DOMINATING SET remains NP-complete even when instances are restricted to bipartite graphs, however, in this case VERTEX COVER is solvable in polynomial time. Consequences to VECTOR DOMINATING SET as a generalization of both are discussed.
\end{abstract}

Keywords: bipartite graph, domination.

2000 Mathematics Subject Classification: 05C35.

For terminology and notation not defined here we refer to $[2,3]$. Given a finite, simple, and undirected graph $G$ without isolated vertices, $V(G)=$ $\{1, \ldots, n\}, E(G), G[V]$, and $d_{i}(G)$ denote its vertex set, its edge set, the subgraph of $G$ induced by $V \subseteq V(G)$, and the degree of $i \in V(G)$ in $G$, respectively. Furthermore, let $\overrightarrow{1}=(1, \ldots, 1) \in R^{n}$ and $\vec{d}(G)=\left(d_{1}(G), \ldots, d_{n}(G)\right)$. Given an integral vector $\vec{k}=\left(k_{1}, \ldots, k_{n}\right)$ with $1 \leq k_{i} \leq d_{i}(G)$ for $i=$ $1, \ldots, n$, a set $D \subseteq V(G)$ is called $\vec{k}$-dominating if $d_{i}(G[V(G) \backslash D]) \leq$ $d_{i}(G)-k_{i}$ for $i \in V(G) \backslash D$. Consider the corresponding decision problem, which was investigated in [4]:

\section{VECTOR DOMINATING SET}

Instance: A graph $G$ on $V(G)=\{1, \ldots, n\}$, an integral vector $\vec{k}=$ $\left(k_{1}, \ldots, k_{n}\right)$ with $1 \leq k_{i} \leq d_{i}(G)$ for $i=1, \ldots, n$, and a positive integer $l$.

Question: Does $G$ contain a $\vec{k}$-dominating set $D$ with $|D| \leq l$ ?

The restriction of VECTOR DOMINATING SET to $\vec{k}=\overrightarrow{1}$ is the decision problem DOMINATING SET, remaining $N P$-complete even when instances are restricted to bipartite graphs [1]. It is easy to see that $D \subseteq V(G)$ is $\vec{d}(G)$ dominating if and only if each edge of $G$ has at least one endvertex in $D$. 
The corresponding decision problem VERTEX COVER is $N P$-complete in general, however, is solvable in polynomial time for bipartite graphs [3]. The question arises in how many components $\vec{k}$ may differ from $\overrightarrow{1}$ or from $\vec{d}(G)$ such that the restriction of VECTOR DOMINATING SET to bipartite graphs remains $N P$-complete or is solvable in polynomial time, respectively. Theorem 1 and Theorem 2 give partial answers to this question.

Theorem 1. Given $0<c<\frac{1}{2}$, the following restriction of VECTOR DOMINATING SET remains $N P$-complete.

Instance: A bipartite graph $G$ on $V(G)=\{1, \ldots, n\}$, an integral vector $\vec{k}=$ $\left(k_{1}, \ldots, k_{n}\right)$ with $1 \leq k_{i} \leq d_{i}(G)$ for $i=1, \ldots, n$ and $\left|\left\{i \in V(G) \mid k_{i}>1\right\}\right|$ $=\lceil c n\rceil$, and a positive integer $l$.

Question: Does $G$ contain a $\vec{k}$-dominating set $D$ with $|D| \leq l$ ?

Theorem 2. Given $c>0$, the following restriction of VECTOR DOMI$N A T I N G$ SET is solvable in polynomial time.

Instance: $A$ bipartite graph $G$ on $V(G)=\{1, \ldots, n\}$, an integral vector $\vec{k}=$ $\left(k_{1}, \ldots, k_{n}\right)$ with $1 \leq k_{i} \leq d_{i}(G)$ for $i=1, \ldots, n$ and $\mid E\left(G\left[\left\{i \in V(G) \mid k_{i}<\right.\right.\right.$ $\left.\left.\left.d_{i}(G)\right\}\right]\right) \mid \leq c \log _{2} n$, and a positive integer $l$.

Question: Does $G$ contain a $\vec{k}$-dominating set $D$ with $|D| \leq l$ ?

Proof of Theorem 1. Given $0<c<\frac{1}{2}$, we shall transform DOMINATING SET to the restriction of VERTEX DOMINATING SET of Theorem 1. Let a graph $H$ on $V(H)=\{1, \ldots, m\}$ and a positive integer $l$ be an instance of DOMINATING SET, the positive integer $r$ be choosen such that $\frac{2 c m}{1-2 c} \leq r<\frac{2 c m}{1-2 c}+1$, hence, $c(2 m+2 r) \leq r<c(2 m+2 r)+$ $(1-2 c)$ implying $r=\lceil c(2 m+2 r)\rceil \geq 1$, and $G$ be constructed with $V(G)=\{1, \ldots, 2 m+2 r\}$ and $E(G)=\{(i, m+j),(j, m+i) \mid(i, j) \in E(H)\}$ $\cup\{(i, m+i),(m+i, 2 m+j),(2 m+j, 2 m+r+j) \mid i=1, \ldots, m, j=1, \ldots, r\}$. Following the ideas in [1], it is easy to see that $G$ is bipartite and that for $D(H) \subseteq V(H), D(G)=\{i+m \mid i \in D(H)\} \cup\{2 m+1, \ldots, 2 m+r\}$ is a $\overrightarrow{1}$-dominating set of $G$ if and only if $D(H)$ is a $\overrightarrow{1}$-dominating set of $H$. Let $\vec{k}=\left(k_{1}, \ldots, k_{2 m+2 r}\right)$ be an arbitrary integral vector with $1 \leq k_{p} \leq d_{p}(G)$ for $p=1, \ldots, 2 m+2 r$, and $k_{p}=1$ if and only if $p \in V(G) \backslash\{2 m+1, \ldots, 2 m+r\}$. Then $\left|\left\{i \in V(G) \mid k_{i}>1\right\}\right|=r=\lceil c|V(G)|\rceil$, and $D(G)$ is even a $\vec{k}$-dominating set of $G$ if $D(H)$ is a $\overrightarrow{1}$-dominating set of $H$. Hence, $H$ contains a $\overrightarrow{1}$-dominating set of cardinality $l$ if and only if $G$ contains a $\vec{k}$-dominating set of cardinality $l+r$. With $|V(G)|=2 m+2 r<2 m+2 c(2 m+2 r)+2(1-2 c)=$ $2|V(H)|+2 c|V(G)|+2(1-2 c)$, hence, $|V(G)|<\frac{2}{1-2 c}|V(H)|+2$ we are done. 
Proof of Theorem 2. For $D \subseteq V(G)$, let $E(D)$ be the set of edges having no endvertex in $D$, and $H(D)$ be the graph arising from $G$ by deleting the edges of $E(D)$. It is easy to see that $D$ is a $\vec{k}$-dominating set of $G$ if and only if $E(D) \subseteq E\left(G\left[\left\{i \in V(G) \mid k_{i}<d_{i}(G)\right\}\right]\right)$, each endvertex $i$ of an edge in $E(D)$ is endvertex of at most $d_{i}(G)-k_{i}$ edges in $E(D)$, and $D$ is a $\vec{d}(H(D))$ dominating set of $H(D)$. Since VERTEX COVER is solvable in polynomial time and the number of sets $E(D)$ is at most $2^{\left|E\left(G\left[\left\{i \in V(G) \mid k_{i}<d_{i}(G)\right\}\right]\right)\right|} \leq n^{c}$ we are done.

Remark. It remains open whether the bounds $c n\left(0<c<\frac{1}{2}\right)$ and $c \log _{2} n$ $(c>0)$ of Theorem 1 and Theorem 2 can be made significantly greater such that VECTOR DOMINATING SET is still NP-complete or solvable in polynomial time, respectively.

\section{References}

[1] G.J. Chang and G.L. Nemhauser, The $k$-domination and $k$-stability problems in sun-free chordal graphs, SIAM J. Algebraic Discrete Methods 5 (1984) 332-345.

[2] R. Diestel, Graph Theory (Springer-Verlag, New York, 2000).

[3] M.R. Garey and D.S. Johnson, Computers and Intractability (W.H. Freeman and Company, San Francisco, 1979).

[4] J. Harant, A. Pruchnewski and M. Voigt, On dominating sets and independent sets of graphs, Combinatorics, Probability and Computing 8 (1999) 547-553.

Received 24 August 2000 